\title{
ON OPERATORS WITH LARGE SELF-COMMUTATORS
}

\author{
GEVORGYAN LEVON
}

Abstract. We study the "size" of self-commutator of Hilbert space operator. Different properties of operators, having large self-commutators are established. Possible values of the numerical radius of such operators are investigated. Two necessary and sufficient conditions of equality $\|A\|=2 w(A)$ are mentioned.

Mathematics subject classification (2000): 47A12, 47B47.

Keywords and phrases: Self-commutator, numerical radius.

\section{REFERENCES}

[1] E. Asplund, V. PTAK, A minimax inequality for operators and a related numerical range, Acta Math., 126 (1971), 53-62.

[2] M. BarraA, M. Boumazgour, Inner derivations and norm equality, Proc. Amer. Math. Soc., 130 (2002), 471-476.

[3] S.S. DRAGOMIR, A survey of some recent inequalities for the norm and numerical radius of operators in Hilbert spaces, Banach J. Math. Anal., 1, 2 (2007), 154-175.

[4] P.R. Halmos, Commutators of operators, Amer. J. Math., 74 (1952), 237-240.

[5] C.R. Putnam, Commutation properties of Hilbert space operators, Springer-Verlag, Berlin, 1967.

[6] J.G. STAMPFLI, The norm of derivation, Pacific. J. Math., 33 (1970), 737-747.

[7] T. YAMAZAKI, Upper and lower bounds of numerical radius and an equality condition, Studia Math., 178 (2007), 83-89. 